\title{
Inhalt
}

\section{A. Einführung}

Dimensionen des Wettbewerbs: Problemstellungen

Karl von Delhaes, Ulrich Fehl

\section{B. Wettbewerb in der Marktsphäre}

\section{Grundlagen}

Wettbewerb als Hypothesentest: Eine evolutorische Konzeption wissenschaffenden Wettbewerbs

Wolfgang Kerber

Wettbewerbspolitische Konzeptionen 79

Herwig Brendel

\section{Wettbewerb über mehrere Marktstufen hinweg}

Die Koordination von Wissen über mehrere Wirtschaftsstufen

Carsten Schreiter

Humanvermögen in evolutionären Wettbewerbsprozessen

Hans-Günter Krüsselberg

Der Wettbewerbszusammenhang zwischen Kapital- und Gütermärkten.

Alfred Schüller

\section{Ausgewählte Märkte mit spezifischen Wettbewerbsproblemen}

Technische Restriktionen als Wettbewerbsproblem - Das Beispiel des Marktes für elektrische Energie

Helmut Gröner

Der Arzneimittelmarkt im Spannungsfeld seiner institutionellen

Umgebung....

Frank Daumann, Peter Oberender

Funktionsbedingungen und Funktionsprobleme des Wettbewerbs im System der deutschen Krankenversicherung.

Kornelia van der Beek, Dieter Cassel 
C. Wettbewerb in anderen Interaktionssphären

I. Theoretische Aspekte

Wettbewerb als ontologische Universalie: Natürliche Arten, wettbewerbliche Interaktionen und Internalisierung.

Carsten Hermann-Pillath

Wettbewerb und Demokratie - Zur Problemstellung einer institutionenökonomischen Ordnungstheorie

Ingo Pies

\section{Befunde der langen und mittleren Frist}

Effiziente Institutionen und wirtschaftlicher „Rückschritt“

Günter Hesse

Der Zusammenhang zwischen der Entstehung und dem Wettbewerb von Ordnungen.

Helmut Leipold

Die Entwicklung der Gewerbeordnung in Deutschland von 1731 bis 1897

Hans-Georg Reuter

Das Zusammenspiel von Wirtschaftsordnung, Unternehmensorganisation und Industrialisierung: Vergleichende Betrachtungen zur Wirtschaftsentwicklung Deutschlands und der USA 1850 - 1914 449

Peter Hertner

\section{Die kurze Frist: Die Bedeutung von Wettbewerb im Transformationsprozeß}

Wettbewerb in verschiedenen Wirtschaftssystemen 471

Leszek Balcerowicz

Instituting Competition as a Spontaneous Element in Transformation

László Czaba

Probleme der sichtbaren Hand bei der Herstellung von

Wettbewerb: Zur Rolle der Treuhandanstalt

Gernot Gutmann, Werner Klein

Deregulierungspolitik und Wettbewerb in Netzindustrien:

Bedeutung und Optionen für osteuropäische Transformationsländer

Cornelius Graack, Paul J.J. Welfens

Sachregister

Autoren und Seminarteilnehmer. 